\section{(2) OPEN ACCESS}

\title{
Comparison of warfarin versus antiplatelet therapy after surgical bioprosthetic aortic valve replacement
}

\author{
Christina Christersson (i) ,' Stefan K James, ${ }^{1,2}$ Lars Lindhagen, ${ }^{2}$ Anders Ahlsson, ${ }^{3,4}$ \\ Örjan Friberg, ${ }^{5}$ Anders Jeppsson, ${ }^{6}$ Elisabeth Ståhle ${ }^{7}$
}

- Additional material is published online only. To view, please visit the journal online (http://dx.doi.org/10.1136/ heartjnl-2019-315453).

1 Department of Medical Sciences, Cardiology, Uppsala University, Uppsala, Sweden ${ }^{2}$ Uppsala Clinical Research Center, Uppsala University, Uppsala, Sweden ${ }^{3}$ Department of Thoracic and Cardiovascular Surgery, Karolinska University Hospital, Stockholm, Sweden ${ }^{4}$ Department of Molecular and Clinical Medicine, Institute of Medicine Sahlgrenska Academy, Gothenburg, Sweden

${ }^{5}$ Department of Cardiothoracic and Vascular Surgery, Örebro University Hospital, Örebro, Sweden

${ }^{6}$ Cardiothoracic Surgery, Sahlgrenska University Hospital, Gothenburg, Sweden ${ }^{7}$ Department of Surgical Sciences Thoracic Surgery, University Hospital Uppsala, Uppsala, Sweden

\section{Correspondence to} Dr Christina Christersson, Cardiology, Medical Sciences, Uppsala, Sweden; christina.christersson@medsci. uu.se

Received 27 May 2019 Revised 19 October 2019 Accepted 25 October 2019 Published Online First 22 November 2019

\section{Check for updates}

(C) Author(s) (or their employer(s)) 2020. Re-use permitted under CC BY-NC. No commercial re-use. See rights and permissions. Published by BMJ.

To cite: Christersson $C$, James SK, Lindhagen $L$, et al. Heart 2020;106:838-844.

\section{ABSTRACT}

Objectives To compare effectiveness of warfarin and antiplatelet exposure regarding both thrombotic and bleeding events, following surgical aortic valve replacement with a biological prosthesis(bioSAVR). Methods The study included all patients in Sweden undergoing a bioSAVR during 2008-2014 who were alive at discharge from the index hospital stay. Exposure was analysed and defined as postdischarge dispension of any antithrombotic pharmaceutical, updated at each following dispensions and categorised as single antiplatelet (SAPT), warfarin, warfarin combined with SAPT, dual antiplatelet (DAPT) or no antithrombotic treatment. Exposure to SAPT was used as comparator. Outcome events were all-cause mortality, ischaemic stroke, haemorrhagic stroke, any thromboembolism and major bleedings. We continuously updated adjustments for comorbidities with any indication for antithrombotic treatment by Cox regression analysis.

Results We identified 9539 patients with bioSAVR (36.8\% women) at median age of 73 years with a mean follow-up of 3.13 years. As compared with SAPT, warfarin alone was associated with a lower incidence of ischaemic stroke (HR 0.49, 95\% Cl 0.35 to 0.70 ) and any thromboembolism (HR 0.75, 95\% Cl 0.60 to 0.94 ) but with no difference in mortality (HR 0.94, $95 \% \mathrm{Cl} 0.78$ to 1.13). The incidence of haemorrhagic stroke (HR 1.94, 95\% Cl 1.07 to 3.51) and major bleeding (HR 1.67, 95\% Cl 1.30 to 2.15) was higher during warfarin exposure. As compared with SAPT, DAPT was not associated with any difference in ischaemic stroke or any thromboembolism. Risk-benefit analyses demonstrated that $2.7(95 \% \mathrm{Cl} 1.0$ to 11.9$)$ of the ischaemic stroke cases could potentially be avoided per every haemorrhagic stroke caused by warfarin exposure instead of SAPT during the first year.

Conclusion In patients discharged after bioSAVR, warfarin exposure as compared with SAPT exposure was associated with lower long-term risk of ischaemic stroke and thromboembolic events, and with a higher incidence of bleeding events but with similar mortality.

\section{INTRODUCTION}

After aortic valve replacement (AVR), patients still experience excess mortality compared with the general population. ${ }^{1}$ The observed excess mortality can be partly attributed to morbidity related to the prosthesis and associated medication. Biological as compared with mechanical prostheses require less intensive antithrombotic therapy and are therefore the first-line choice in most patients undergoing surgical AVR.

The reported incidence of long-term thromboembolic events after aortic valve surgery with a bioprosthesis (bioSAVR) vary considerably, and generalisation of historical reports to current AVR cohorts must be done with caution. ${ }^{23}$ Patient characteristics have changed over the years with higher mean age, more atrial fibrillation (AF), concomitant coronary disease and comorbidities that contribute to increased risk of thromboembolic events yet with a lower 30 -day mortality. ${ }^{34}$ There is a substantial amount of data supporting long-term oral anticoagulant treatment (OAC) after valve intervention in patients with $\mathrm{AF}$ but whether the majority of patients without prior AF benefit from OAC treatment is not established. ${ }^{56}$ In the absence of other indications for OAC, current guidelines recommend oral antithrombotic treatment with aspirin or warfarin for 3 months after bioSAVR. ${ }^{56}$ This recommendation is based on a low level of evidence and should be regarded as expert opinions only. ${ }^{56}$

The occurrence of leaflet thrombosis and immobility in bioprostheses with the potential influence on the risk of ischaemic stroke has been increasingly recognised after both transcatheter AVR (TAVI) and bioSAVR. ${ }^{78}$ However, the true incidence is unclear and the clinical relevance of subclinical leaflet thrombosis is not well established. The incidence of thromboembolic events during the first 3 months after discharge is low and in some series the majority of valve thrombosis cases after SAVR occur $>1$ year after implantation. ${ }^{89}$ Altogether, these data suggest that it might be beneficial to prolong the duration and/or intensity of antithrombotic treatment. On the contrary, bleeding risk increases with longer duration. In summary, there are conflicting opinions on the optimal antithrombotic treatment, including strategy and duration of treatment after intervention with a biological prosthesis. ${ }^{9-12}$ This study therefore aimed to evaluate the effectiveness of different antithrombotic therapies to identify the antithrombotic regimen that yields highest possible balance between thrombotic and bleeding risks following bioSAVR.

\section{METHODS}

\section{Study population and data sources}

Surgical AVR is performed at eight centres in Sweden. All patients undergoing cardiac surgery are continuously included in the Swedish Web system for Enhancement and Development of 
Evidence-based care in Heart disease Evaluated According to Recommended Therapies (SWEDEHEART). ${ }^{13}$ The present study cohort included all patients undergoing a bioSAVR, with or without coronary artery bypass grafting, between 1 January 2008 and 31 December 2014 and who were alive at discharge from the index intervention. The bioSAVR group included all types of biological prosthesis. Patients receiving more than one valve prosthesis $(n=332)$ and patients treated with a mechanical prosthesis $(n=2447)$ were not included. In addition, patients with missing information about previous coronary intervention or left ventricular ejection fraction (LVEF) at index intervention $(n=55)$ were excluded. All patients admitted for bioSAVR are informed of the inclusion in the SWEDEHEART according to ethical approval, and no written informed consent are obtained.

\section{Data collection}

The SWEDEHEART registry contains detailed information on the procedures and concomitant diseases. Baseline information from SWEDEHEART was enriched with information from the National Patient Register (NPR), which includes the diagnosis codes (International Code of Disease, 10th Revision (ICD-10)) of all hospital admissions in Sweden since $1987 .{ }^{14}$ Linkage was based on the unique 10-digit personal identification number assigned to all Swedish residents at birth or immigration. The National Board of Health and Welfare merged the registries.

All patients were followed through computerised linkage between the database and the updated census register, the Swedish Cause of Death Register and the NPR, all managed by the National Board of Health and Welfare. The start date was 1 day after discharge from the index intervention. The study cohort was followed until death or the end of follow-up (31 December 2014), whichever occurred first.

\section{Baseline information and comorbidities}

Information on baseline characteristics and previously diagnosed comorbidities occurring up to 3 years before the valve intervention were defined as hospital admission due to any of the comorbidities as the primary diagnosis in the NPR or collected from the SWEDEHEART registry. Predefined comorbidities at baseline were diabetes, hypertension, congestive heart failure (CHF), AF, myocardial infarction (MI), any thromboembolism (ie, ischaemic stroke, systemic embolism, pulmonary embolism and venous thromboembolism) and major bleeding (ie, haemorrhagic stroke and hospitalisation for other bleeding event). Comorbidities were continuously updated during the follow-up and included in the adjusted models. The ICD-10 was applied to identify comorbidities and outcome events (online supplementary eTable 1).

\section{Exposure to oral antithrombotic treatment}

Information on the dispension of oral antiplatelet and anticoagulant treatment was collected by computerised linkage with the Dispensed Drug Register. The register contains information on every prescription and dispension of drugs in every pharmacy in Sweden. The patient was considered exposed to the corresponding dispensed antithrombotic treatment for 120 days after each dispension, if no new dispension occurred. Information on dispension was continuously updated, and patients could be exposed to different pharmaceutical treatment during follow-up and patients changed groups every time a new class of antithrombotic agent was dispensed. The constructed variable regarding exposure to oral antithrombotic treatment at any given time point was categorised into five exposure groups: 1) single antiplatelet treatment (SAPT, either aspirin or P2Y $\mathrm{Y}_{12}$ inhibitor), 2) warfarin, 3) warfarin plus a SAPT or a dual antiplatelet treatment (DAPT), 4) DAPT (aspirin and P2Y 12 inhibitor) only and 5) no antithrombotic treatment. Information on dispension of non-vitamin $\mathrm{K}$ antagonist oral anticoagulant drugs was collected separately, and not included in further analyses.

\section{Outcome events}

Outcome events after discharge from the index surgical procedure were all-cause mortality, new hospitalisation for ischaemic stroke, any thromboembolism, haemorrhagic stroke and major bleeding.

\section{Statistical methods}

Categorical variables were expressed as frequencies and percentages and continuous variables as median and IQRs (Q1-Q3).

Exposure to a pharmaceutical agent was assessed and updated at every dispension. Consequently, each patient could be exposed to different pharmaceutical categories during follow-up. The sum of periods that patients were exposed to each pharmaceutical category was computed and presented in person-years (PY) for each pharmaceutical category and an incidence rate/100 PY was calculated for each outcome event, started at discharge from the index intervention.

Kaplan-Meier curves for time-dependent exposure, also known as Simon-Makuch curves, were created by restarting patients after a treatment switch. ${ }^{15}$

The association between outcome events and baseline characteristics and continuously updated comorbidities (denominated prior AF, prior thromboembolism, prior major bleeding, prior MI and prior coronary intervention) were explored in unadjusted Cox regression analyses for each variable.

The associations between oral antithrombotic exposure groups and repeated outcome events were analysed using multivariable Cox regression models. To account for data dependency due to recurrent events, robust Huber-White SEs were computed. ${ }^{16} \mathrm{~A}$ crude model that includes only antithrombotic exposure as a time-dependent variable was performed for each outcome event: all-cause mortality, ischaemic stroke, any thromboembolism, haemorrhagic stroke and major bleeding. In addition, adjusted models were fitted, included age, sex, LVEF, diabetes, hypertension, $\mathrm{CHF}$ and the continuously updated comorbidities; prior $\mathrm{AF}$, prior thromboembolism (ie, ischaemic stroke, systemic embolism, pulmonary embolism and venous thromboembolism), prior major bleeding, (ie, haemorrhagic stroke and hospitalisation for other bleeding event), prior MI, prior coronary intervention and coronary intervention at index valve replacement.

The results of unadjusted and fully adjusted Cox analysis were presented as HRs with 95\% CIs. The proportional hazard assumptions were checked using Schoenfeld residuals.

Interactions between antithrombotic treatment exposure and age or prior AF was tested for using an interaction term in the multivariable Cox model.

A sensitivity analysis was performed of patients without prior $\mathrm{AF}$ at the index intervention. In a risk-benefit analysis, the number of prevented ischaemic strokes per caused haemorrhagic stroke due to warfarin treatment instead of SAPT were estimated. This analysis was performed by estimating absolutes risks, separately for each outcome and treatment, using the baseline hazard from the adjusted Cox model as described above. This was done for a random sample of 200 patients, for computer performance reasons, keeping their comorbidities fixed at the baseline values and synthetically setting the antithrombotic treatment to either 
Table 1 Baseline characteristics expressed as frequencies and percentages if not otherwise stated

\begin{tabular}{|c|c|}
\hline & bioSAVR $n=9539$ \\
\hline $\begin{array}{l}\text { Age at index intervention median (Q1- } \\
\text { Q3) }\end{array}$ & $73(67-78)$ \\
\hline \multicolumn{2}{|l|}{ Sex } \\
\hline Female & $3509(36.8 \%)$ \\
\hline Male & $6030(63.2 \%)$ \\
\hline \multicolumn{2}{|l|}{ LVEF } \\
\hline$>0.50$ & $6991(73.1 \%)$ \\
\hline $0.30-0.50$ & $2072(21.7 \%)$ \\
\hline$<0.30$ & $496(5.2 \%)$ \\
\hline $\begin{array}{l}\text { Coronary intervention at index } \\
\text { intervention* }\end{array}$ & $3285(34.4 \%)$ \\
\hline \multicolumn{2}{|l|}{ Medical history } \\
\hline Diabetes & $1833(19.2 \%)$ \\
\hline Hypertension & $3736(39.2 \%)$ \\
\hline Ischaemic stroke & $819(8.6 \%)$ \\
\hline Systemic embolism & $314(3.3 \%)$ \\
\hline $\begin{array}{l}\text { Pulmonary embolism and venous } \\
\text { thromboembolism }\end{array}$ & $684(7.2 \%)$ \\
\hline Haemorrhagic stroke & $36(0.4 \%)$ \\
\hline Other bleeding event & $355(3.7 \%)$ \\
\hline Myocardial infarction & $1264(13.3 \%)$ \\
\hline Peripheral artery disease & $612(6.4 \%)$ \\
\hline Heart failure & $1234(12.9 \%)$ \\
\hline Atrial fibrillation & $1454(15.2 \%)$ \\
\hline Previous thoracic surgery & $470(4.9 \%)$ \\
\hline $\begin{array}{l}\text { Previous percutaneous coronary } \\
\text { intervention }\end{array}$ & $1105(11.6 \%)$ \\
\hline
\end{tabular}

warfarin or SAPT. Finally, these risks were averaged over the 200 patients, and the ratio between the risk differences (SAPT vs warfarin) was computed. CIs were computed based on 2500 bootstrap replicates.

\section{Patient and public involvement}

The patients were not involved in the present study.

\section{RESULTS}

\section{Clinical characteristics}

The study cohort consisted of 9539 patients who had received a surgical biological prosthetic valve in the aortic position. The median age at index intervention was 73 (range 67-78) years and $3509(36.8 \%)$ were females. Prior to intervention, 819 (8.6\%) patients had a history of ischaemic stroke and $36(0.4 \%)$ patients had suffered from a haemorrhagic stroke (table 1). At baseline, 1454 (15.2\%) patients had experienced AF prior to intervention and another 1064 experienced AF during follow-up. Few patients experienced MI or coronary intervention during follow-up (data not shown).

\section{Outcome events during follow-up}

No patient was lost from follow-up. During a mean follow-up of 3.13 years (median 2.99, maximum 6.97 years), 1157 (12.2\%) patients died (3.92/100 PY). In total, there were 895 thromboembolic events (3.04/100 PY). Out of these, 405 (45\%) were an ischaemic stroke (1.37/100 PY). There were in total 573 major bleeding events (1.94/100 PY) and of these 129 (23\%) were a haemorrhagic stroke (0.44/100 PY). Of the outcome events 128 deaths, 157 thromboembolic events and 138 major bleeding events occurred during the first 3 months after discharge.

\section{The association of outcome events with comorbidities}

Comorbidities associated with outcome events are presented in online supplementary eTable 2 . The most important risk factors of ischaemic stroke and haemorrhagic stroke were prior occurrence of a thromboembolic (HR 3.89, 95\% CI 3.12 to 4.86 ) or a bleeding event (HR 8.86, 95\% CI 6.35 to 12.37), (online supplementary eTable 2 ), respectively.

\section{Antithrombotic exposure}

During the total follow-up (29487 PY), the proportion of exposure in relation to the total person-time to SAPT was $52.7 \%$, to warfarin $19.3 \%$, to warfarin in combination with SAPT 5.8\% and to DAPT $1.0 \%$. Patients were without antithrombotic treatment during $21.2 \%$ of the total person-time (online supplementary eTable 3). During the first 3 months after discharge, the proportion of exposure to SAPT was $32.6 \%$, to warfarin $31.7 \%$, to warfarin in combination with SAPT $27.1 \%$ and to DAPT $1.8 \%$.

\section{The association of outcome events to antithrombotic treatment}

The number of outcome events and the corresponding incidence rates of death, ischaemic stroke, any thromboembolism, haemorrhagic stroke and any bleeding by antithrombotic treatment exposure are presented in table 2.

\section{All-cause mortality}

The lowest mortality rate was noted during exposure to SAPT (3.04/100 PY) and the highest during periods without oral antithrombotic treatment (6.10/100 PY) (table 2, figure 1A). Warfarin exposure was not associated with higher mortality as compared with SAPT (HR 0.94, 95\% CI 0.78 to 1.13 ) (figure 2A). An additional antiplatelet drug (DAPT 5.74/100 PY) did not influence

Table 2 Antithrombotic exposure and outcome events during follow-up

\begin{tabular}{|c|c|c|c|c|c|}
\hline & $\begin{array}{l}\text { Single antiplatelet } \\
\text { treatment }\end{array}$ & Warfarin treatment & Warfarin+SAPT & $\begin{array}{l}\text { Dual antiplatelet } \\
\text { treatment }\end{array}$ & $\begin{array}{l}\text { No oral antithrombotic } \\
\text { treatment }\end{array}$ \\
\hline All-cause mortality* & 473 (155.5) 3.04 & $212(56.9) 3.72$ & $74(17.1) 4.34$ & $17(3.0) 5.74$ & $381(62.4) 6.10$ \\
\hline Ischaemic stroke* & 215 (155.5) 1.38 & $57(56.9) 1.00$ & $29(17.1) 1.70$ & $6(3.0) 2.03$ & $98(62.4) 1.57$ \\
\hline Any thromboembolism* & $442(155.5) 2.84$ & $163(56.9) 2.86$ & 71 (17.1) 4.16 & $12(3.0) 4.05$ & $207(62.4) 3.32$ \\
\hline Haemorrhagic stroke* & 42 (155.5) 0.27 & $42(56.9) 0.74$ & $21(17.1) 1.23$ & $1(3.0) 0.34$ & $23(62.4) 0.37$ \\
\hline Major bleeding* & 207 (155.5) 1.33 & $172(56.7) 3.02$ & $84(17.1) 4.93$ & $4(3.0) 1.35$ & $106(62.4) 1.70$ \\
\hline
\end{tabular}

\footnotetext{
*Expressed as frequencies (person-time) incidence rate/100 patient-years. 
A

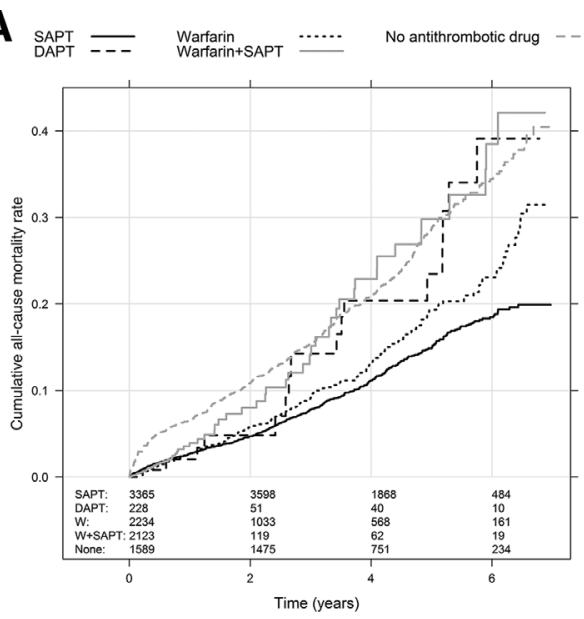

B
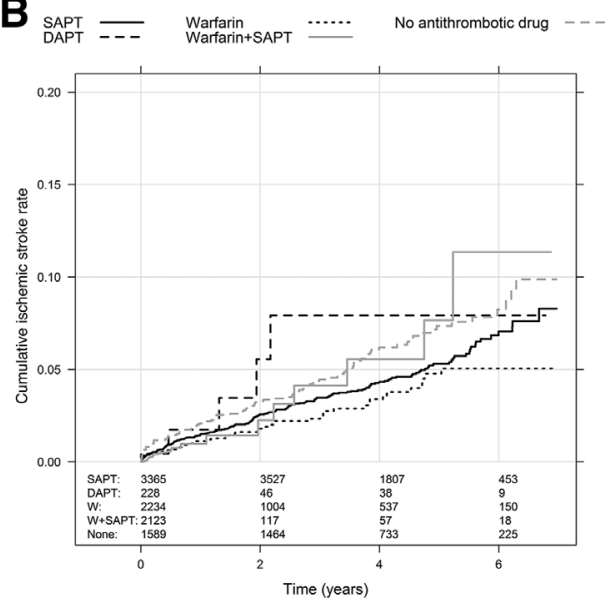

Figure 1 Incidence of outcome events by different antithrombotic exposures Kaplan-Meier estimates for (A) all-cause mortality, (B) ischaemic stroke and (C) haemorrhagic stroke during follow-up. The lines represent different antithrombotic exposures: single antiplatelet treatment (SAPT), warfarin, W+SAPT, dual antiplatelet treatment (DAPT) and no oral antithrombotic treatment.

mortality (HR 1.32, 95\% CI 0.81 to 2.15 ) as compared with SAPT alone (figure 2A). The overall risk of mortality was higher (HR 2.14 , 95\% CI 1.85 to 2.49 ) during periods without antithrombotic treatment as compared with SAPT (figure 2A). Similar results were found when the group with AF prior to intervention were excluded from the analyses (online supplementary eFigure 3). No interaction was found between antithrombotic treatment and age $(\mathrm{p}=0.11)$ and prior $\mathrm{AF}(\mathrm{p}=0.065)$.

\section{Ischaemic stroke and any thromboembolism}

During SAPT exposure, the incidence rate was $1.38 / 100$ PY for ischaemic stroke and 2.84/100 PY for any thromboembolism (table 2, figure $1 \mathrm{~B}$, online supplementary eFigure 1 ). The incidence rate observed during warfarin exposure indicated a somewhat lower incidence of ischaemic stroke (1.00/100 PY) but not for any thromboembolism (2.86/100 PY) (table 2). In adjusted analysis warfarin exposure gave a lower incidence of ischaemic stroke (HR $0.49,95 \%$ CI 0.35 to 0.70 ) and any thromboembolism (HR 0.75, 95\% CI 0.60 to 0.94) as compared with SAPT (figure 2B, online supplementary eFigure 2). There were no differences between DAPT and SAPT exposure (figure 2B, online supplementary eFigure 2). During time periods without antithrombotic exposure, the incidence of ischaemic stroke was similar, but a higher incidence of any thromboembolism was observed (HR 1.31, 95\% CI 1.09 to 1.58 ) compared with SAPT (online supplementary eFigure 2). Similar results were found when the group with AF prior to intervention were excluded from the analyses (online supplementary eFigure 3 ).

No interaction was found between antithrombotic treatment and age regarding the incidence of ischaemic stroke $(p=0.12)$ but concerning any thromboembolism $(p=0.004)$. Warfarin was associated with lower incidence of any thromboembolism in older age groups. No interactions were found between antithrombotic treatment and prior AF regarding the incidence of ischaemic stroke $(p=0.36)$ or any thromboembolism $(p=0.64)$.

\section{Haemorrhagic stroke and major bleeding}

During SAPT exposure, the incidence rate was 0.27/100 PY for haemorrhagic stroke and 1.33/100 PY for major bleeding (table 2, figure 1C, online supplementary eFigure 1). During warfarin exposure, the incidence rate was $0.74 / 100$ PY for haemorrhagic stroke and 3.02/100 PY for major bleeding (table 2). After multivariable adjustment, warfarin exposure was associated with a higher incidence of haemorrhagic stroke (HR 1.94, 95\% CI 1.07 to 3.51 ) and major bleeding (HR 1.67, 95\% CI 1.30 to 2.15 ) as compared with SAPT (figure $2 \mathrm{C}$, online supplementary eFigure 2). The number of haemorrhagic stroke hospitalisations during DAPT exposure was low $(n=1)$. No difference was found between DAPT or no antithrombotic treatment and SAPT exposure regarding major bleeding (online supplementary 
A)

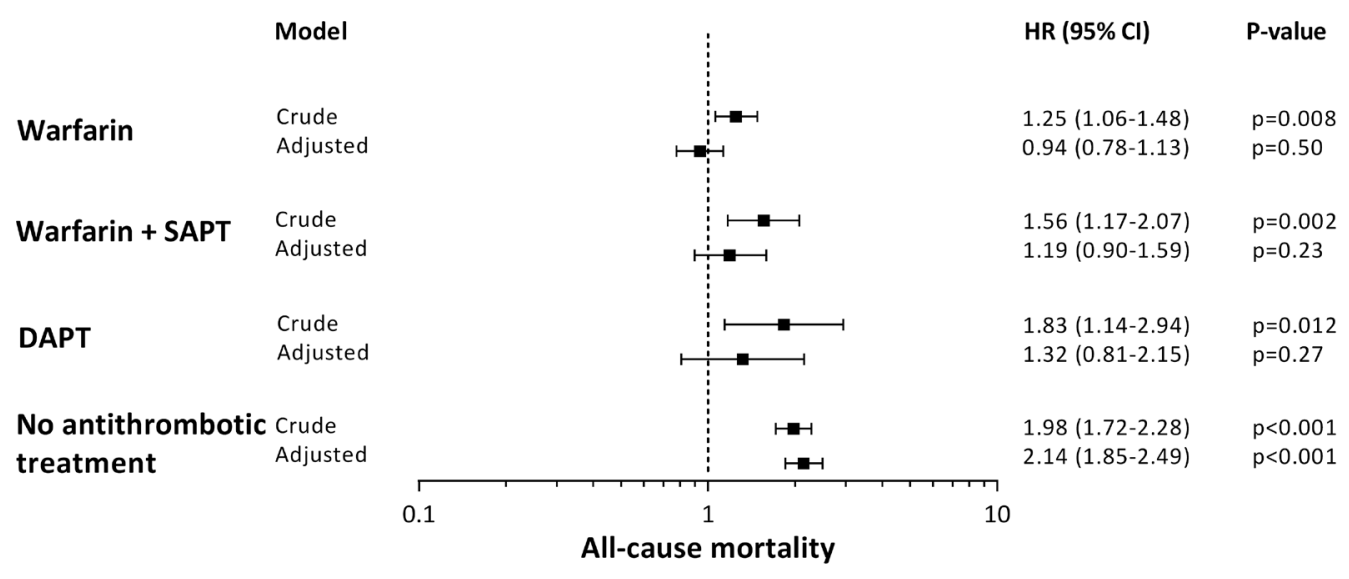

B)

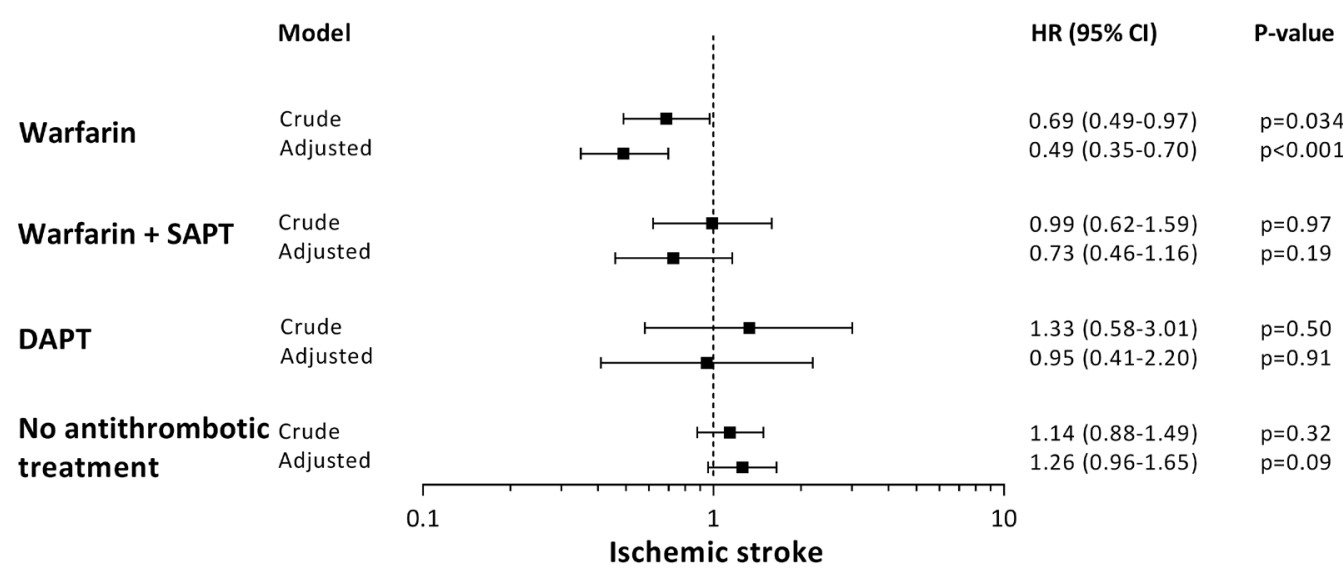

C)

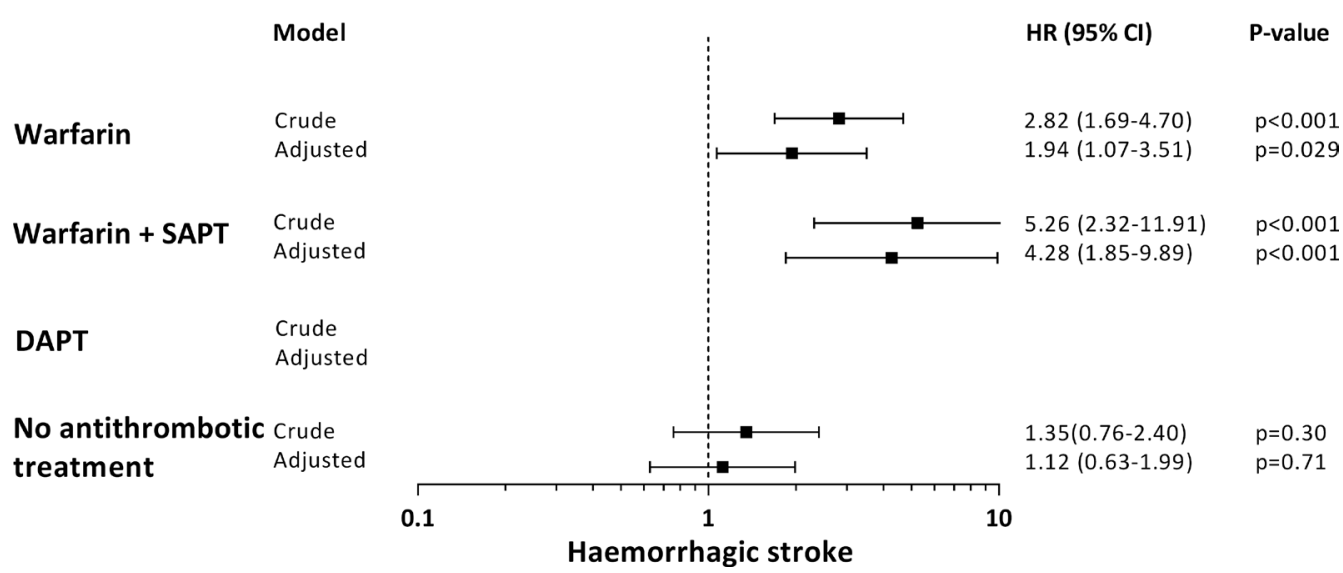

Figure 2 The relative risk of outcome events by different antithrombotic exposures relative to SAPT forest plots describing the relative risk of (A) all-cause mortality, (B) ischaemic stroke and (C) haemorrhagic stroke by warfarin, warfarin+single antiplatelet treatment (SAPT), dual antiplatelet treatment (DAPT) and no antithrombotic treatment relative to SAPT in a crude model. The adjusted model included adjustment for age, sex, left ventricular ejection fraction $(<0.30,0.30-0.50,>0.50)$, diabetes, hypertension, heart failure and the continuously updated comorbidities; prior atrial fibrillation, prior thromboembolism (ie, ischaemic stroke, systemic embolism, pulmonary embolism and venous thromboembolism), prior major bleeding (ie, haemorrhagic stroke and hospitalisation for other bleeding), prior myocardial infarction, prior coronary intervention and coronary intervention at index valve replacement. The number of haemorrhagic strokes were low during exposure to DAPT $(n=1)$ and no relative risk analysis was performed (C).

eFigure 2). Similar results were found when the group with AF prior to intervention was excluded from the analyses (online supplementary eFigure 4).

There were no interactions for antithrombotic treatment and age regarding haemorrhagic stroke $(\mathrm{p}=0.90)$ or major bleeding $(p=0.60)$. No interactions were found between antithrombotic treatment and prior AF regarding the incidence of haemorrhagic stroke $(\mathrm{p}=0.83)$ but concerning major bleeding $(\mathrm{p}<0.001)$.

\section{Risk-benefit of antithrombotic treatment}

Risk-benefit analyses demonstrated that 2.7 (95\% CI 1.0 to 11.9) of the ischaemic strokes could potentially be avoided 
per every haemorrhagic stroke caused by warfarin, if patients were exposed to warfarin instead of SAPT during the first year. Similar results were found after 3 years, 2.0 (95\% CI. 0.8 to 9.0).

\section{DISCUSSION}

After surgical AVR with a biological prosthesis, warfarin treatment was associated with a lower incidence of ischaemic stroke and any thromboembolism without higher mortality as compared with SAPT during long-term follow-up in this study of actual pharmaceutical exposure analysing nationwide complete registries. Furthermore, no tendency towards lower incidence of ischaemic stroke associated with DAPT as compared with SAPT was observed. As expected, exposure to increased intensity of antithrombotic treatment (ie, warfarin) was associated with a higher incidence of haemorrhagic stroke.

Biological aortic prosthesis exposes the patient to increased risk of thromboembolic events. ${ }^{17} 18$ This concern has been proposed to be of greater clinical relevance early after an intervention as reflected in current recommendations of antithrombotic treatment for 3 months after biological valve surgery. ${ }^{5}$ However, the number of studies supporting this position is limited, and it is increasingly being questioned. ${ }^{2}{ }^{218} \mathrm{~A}$ prospective, non-randomised study comparing aspirin with warfarin for 3 months starting 1 day after surgery found no significant differences between the two treatments. ${ }^{11}$ Results from retrospective studies are conflicting, describing similar effects of aspirin and warfarin or an increased risk of thromboembolic events after cessation of warfarin during the first 6 months postintervention. ${ }^{9}{ }^{12}$ In our study, ongoing treatment with warfarin, up to 3 years after intervention, was associated with lower incidence of ischaemic stroke, representing a large proportion of all thromboembolic events, compared with SAPT.

In recent years, the issue of valve thrombosis with and without leaflet immobility has been highlighted and proposed to be important for thromboembolic events and structural valve failure. ${ }^{19}$ In these studies, most valve thrombosis events occurred $>1$ year after the intervention, which may indicate a potential benefit of extended antithrombotic therapy. ${ }^{8}{ }^{17}$ Prostheses evaluated by CT exhibits a larger proportion of subclinical leaflet thrombosis than previously known and has been described after both bioSAVR and TAVI, and OAC has been shown to improve valve motion. ${ }^{20}$ The importance of these findings remains to be fully comprehended but an association between leaflet thrombosis and stroke or transient ischaemic attack is plausible and has previously been described. ${ }^{7}$

In our large, nationwide, well-described and unselected cohort, $15 \%$ of the patients had AF as comorbidity before intervention, and another 6\% experienced AF during follow-up, a condition that has previously been shown to be strongly associated with mortality and stroke in AVR. ${ }^{21}{ }^{22}$ For these patients, therapy with OAC is considered a relatively safe option. ${ }^{35}$ In the present study, representing a bioSAVR cohort, the association between warfarin use and ischaemic stroke was not significantly modulated by prior AF. Therefore, our results support strengthening the indication for $\mathrm{OAC}$ regardless of $\mathrm{AF}$ as comorbidity or AF during follow-up. Moreover, warfarin exposure reduced the total incidence of thromboembolic events, and the association with OAC exposure remained after adjustment for cardiovascular risk factors, suggesting that conclusions about benefit in this study could be drawn for the entire cohort. However, further studies are warranted to establish the patient groups with the highest benefit of OAC.
Overall warfarin exposure was not related to higher mortality. However, as compared with SAPT, warfarin was associated with a higher risk of haemorrhagic stroke. ${ }^{23}$ In the risk-benefit analysis, 2.7 ischaemic strokes could be prevented for each haemorrhagic stroke caused by warfarin. Considering the approximately 2000 patients undergoing bioSAVR in Sweden every year, a substantial number of ischaemic stroke cases might be avoided if patients were consistently shifted to warfarin therapy. There was no interaction between antithrombotic treatment and age on clinical outcomes. Since the incidence of ischaemic stroke increases with increasing age, the relative efficiency obtained by warfarin exposure in the elderly results in a more prominent absolute benefit. Notably, we found no tendency towards increased bleeding risk associated with warfarin in the elderly.

This study confirms an association between warfarin exposure with any major bleeding, of which haemorrhagic stroke only represented a minority of such events. ${ }^{24}$ The most important risk factor for a major bleeding event during follow-up was a prior bleeding emphasising the importance of risk stratification of bleeding in bioSAVR.

There was no indication for benefit of DAPT when compared with SAPT in the current bioSAVR cohort, even though exposure to DAPT was low. These results are consistent with previously reported data in TAVI. ${ }^{10}$ Prospective randomised trials comparing DAPT and SAPT after bioSAVR are warranted.

\section{Limitations}

This study is based on national registries that enable generalisation to European surgical AVR populations. ${ }^{13}$ The SWEDEHEART registry has complete national unselected enrolment of patients undergoing bioSAVR and has been subjected to onsite monitoring and validation and shown to produce high levels of agreement between the registry and available data in the electronic health records. ${ }^{13}$ The NPR contains data on all hospital admissions in Sweden since 1987, with a validity of cardiovascular diagnosis approaching 95\%. ${ }^{14}$ Another limitation concerns the potential impact that an unmeasured confounder could have on the study; however, our study included most clinically relevant information that could explain the association between these pharmaceuticals and outcome. The definition of drug exposure after the last dispension of the antithrombotic drug indicates that actual intake is not guaranteed. ${ }^{25}$ Furthermore, we had no information on the international normalised ratio of the patients prescribed warfarin. However, the anticoagulant treatment in Sweden is generally of high quality. ${ }^{26}$ Pharmaceuticals are involved in a comprehensive reimbursement programme in Sweden, and usage of pharmaceuticals outside this programme can be expected to be marginal also regarding aspirin. The optimal duration of antithrombotic treatment after bioSAVR is not defined in the present study and has to be further evaluated. Despite the limitations noted, our approach, which included a continuous update of pharmaceutical exposure during all follow-ups, should offer an accurate assessment of outcome associated with exposure to prosthesis and related medication. Finally, our study has to be interpreted with caution because of inherent limitations to all observational studies related to confounding by indication.

\section{CONCLUSIONS}

Compared with SAPT, antithrombotic treatment with warfarin after surgical intervention with an aortic biological prosthesis was associated with a lower incidence of ischaemic stroke 


\section{Key questions}

\section{What is already known on this subject?}

- Guidelines for antithrombotic treatment after bioSAVR in patients without other indication for oral anticoagulant treatment recommend aspirin or warfarin for 3 months, based on low level of evidence.

- The risk of leaflet thrombosis and thromboembolism is not limited to the first 3 months.

\section{What might this study add?}

- After bioSAVR, exposure to warfarin treatment was associated with a lower incidence of ischaemic stroke without higher mortality as compared with single antiplatelet therapy (SAPT) during long-term follow-up.

- Risk-benefit analyses demonstrated that 2.7 (95\% CI 1.0 to 11.9) of the ischaemic stroke cases could potentially be avoided per every haemorrhagic stroke caused by warfarin exposure instead of SAPT during the first year.

\section{How might this impact on clinical practice?}

- The association between warfarin exposure and ischaemic stroke was not significantly modulated by prior atrial fibrillation.

- Our results support strengthening the indication for oral anticoagulant treatment in all patients with bioSAVR and underlies the need of thorough risk-benefit analysis regarding antithrombotic treatment not limited to the first 3 months.

and any thromboembolism during long-term follow-up. Warfarin was not associated with higher mortality but with a higher incidence of haemorrhagic stroke. DAPT was not superior to SAPT regarding the risk of ischaemic stroke or any thromboembolism.

Contributors CC, SKJ and ES contributed substantially to the design of the present study. LL performed the statistical analyses. CC and ES provided the first draft of the manuscript. All coauthors participated in the interpretation of data and critically revised the manuscript. All authors have approved the final version of the manuscript. CC, ES and LL had full access to the database and take responsibility for the integrity and of the data and the data analyses.

Funding This work was supported by the European Society of Cardiology, ESC grant for Medical Research Innovation sponsored by Boehringer Ingelheim.

Competing interests $\mathrm{CC}$ has received lecture fees from Boehringer Ingelheim and Bristol Myers Squibb. SKJ has received institutional research grants from AstraZeneca, Jansen and lecture fees from AstraZeneca, Jansen and Bayer. AJ has received support for investigator-initiated studies, consultant fees and speaker's honoraria from AstraZeneca and speaker's honoraria from Boehringer Ingelheim. LL, AA, ÖF and ES have no competing interests to declare.

Patient consent for publication Not required.

Ethics approval The study was approved by the local ethics committee (log no. 2014/518) and in compliance with the regulations of the Declaration of Helsinki.

Provenance and peer review Not commissioned; externally peer reviewed.

Data availability statement No data are available

Open access This is an open access article distributed in accordance with the Creative Commons Attribution Non Commercial (CC BY-NC 4.0) license, which permits others to distribute, remix, adapt, build upon this work non-commercially, and license their derivative works on different terms, provided the original work is properly cited, appropriate credit is given, any changes made indicated, and the use is non-commercial. See: http://creativecommons.org/licenses/by-nc/4.0/.

\section{ORCID iD}

Christina Christersson http://orcid.org/0000-0001-9116-8084

\section{REFERENCES}

1 Kvidal P, Bergström PR, Hörte L-G, et al. Observed and relative survival after aortic valve replacement. J Am Coll Cardiol 2000;35:747-56.

2 Babin-Ebell J, Schmidt W, Eigel P, et al. Aortic bioprosthesis without early anticoagulation-risk of thromboembolism. Thorac Cardiovasc Surg 1995:43:212-4

3 Cohn LH, Collins JJ, Rizzo RJ, et al. Twenty-Year follow-up of the Hancock modified orifice porcine aortic valve. Ann Thorac Surg 1998;66:S30-4. 4 T J. SWEDEHEART Annual report. 2017.

4 J T. SWEDEHEART annual report, 2017.

5 Baumgartner $\mathrm{H}$, Falk V, Bax JJ, et al. ESC/EACTS guidelines for the management of valvular heart disease. Eur Heart J 2017:2017:2739-91.

6 Nishimura RA, Otto CM, Bonow RO, et al. AHA/ACC focused update of the 2014 AHA/ACC guideline for the management of patients with valvular heart disease: a report of the American College of Cardiology/American heart association Task force on clinical practice guidelines. Circulation 2017;2017:e1159-95.

7 Chakravarty T, Søndergaard L, Friedman J, et al. Subclinical leaflet thrombosis in surgical and transcatheter bioprosthetic aortic valves: an observational study. The Lancet 2017:389:2383-92.

8 Egbe AC, Pislaru SV, Pellikka PA, et al. Bioprosthetic valve thrombosis versus structural failure: clinical and echocardiographic predictors. J Am Coll Cardio/ 2015:66:2285-94.

9 Brennan JM, Edwards FH, Zhao Y, et al. Early anticoagulation of bioprosthetic aortic valves in older patients: results from the Society of thoracic surgeons adult cardiac surgery national database. J Am Coll Cardiol 2012;60:971-7.

10 Gandhi S, Schwalm J-DR, Velianou JL, et al. Comparison of Dual-antiplatelet therapy to Mono-antiplatelet therapy after transcatheter aortic valve implantation: systematic review and meta-analysis. Canadian Journal of Cardiology 2015;31:775-84.

11 Gherli T, Colli A, Fragnito C, et al. Comparing warfarin with aspirin after biological aortic valve replacement. Circulation 2004;110:496-500.

12 Mérie C, Køber L, Skov Olsen P, et al. Association of warfarin therapy duration after bioprosthetic aortic valve replacement with risk of mortality, thromboembolic complications, and bleeding. JAMA 2012:308:2118-25.

13 Jernberg T, Attebring MF, Hambraeus K, et al. The Swedish Web-system for enhancement and development of evidence-based care in heart disease evaluated according to recommended therapies (SWEDEHEART). Heart 2010;96:1617-21.

14 Ludvigsson JF, Andersson E, Ekbom A, et al. External review and validation of the Swedish national inpatient register. BMC Public Health 2011:11:450.

15 Simon R, Makuch RW. A non-parametric graphical representation of the relationship between survival and the occurrence of an event: application to Responder versus non-responder bias. Stat Med 1984;3:35-44.

16 Kleinbaum D, Klein M. Survival analysis. 3rd edition. Springer, 2010

17 Brown ML, Park SJ, Sundt TM, et al. Early thrombosis risk in patients with biologic valves in the aortic position. J Thorac Cardiovasc Surg 2012;144:108-11.

18 Heras M, Chesebro JH, Fuster V, et al. High risk of thromboemboli early after bioprosthetic cardiac valve replacement. J Am Coll Cardiol 1995:25:1111-9.

19 Holmes DR, Mack MJ. Aortic valve bioprostheses: leaflet immobility and valve thrombosis. Circulation 2017:135:1749-56.

20 Makkar RR, Fontana G, Jilaihawi $\mathrm{H}$, et al. Possible subclinical leaflet thrombosis in bioprosthetic aortic valves. N Engl J Med 2015;373:2015-24.

21 Wang TKM, Ramanathan T, Choi DH-M, et al. Preoperative atrial fibrillation predicts mortality and morbidity after aortic valve replacement. Interact Cardiovasc Thorac Surg 2014;19:218-22.

22 Yankelson L, Steinvil A, Gershovitz L, et al. Atrial fibrillation, stroke, and mortality rates after transcatheter aortic valve implantation. Am J Cardiol 2014;114:1861-6.

23 van Walraven C, Hart RG, Singer DE, et al. Oral anticoagulants vs aspirin in nonvalvular atrial fibrillation: an individual patient meta-analysis. JAMA 2002;288:2441-8.

24 Vazquez FJ, Gonzalez JP, LeGal G, et al. Risk of major bleeding in patients receiving vitamin $\mathrm{K}$ antagonists or low doses of aspirin. A systematic review and meta-analysis. Thromb Res 2016;138:1-6.

25 Evans M, Carrero J-J, Szummer K, et al. Angiotensin-Converting Enzyme Inhibitors and Angiotensin Receptor Blockers in Myocardial Infarction Patients With Renal Dysfunction. J Am Coll Cardiol 2016:67:1687-97.

26 Björck F, Sandén P, Renlund $H$, et al. Warfarin treatment quality is consistently high in both anticoagulation clinics and primary care setting in Sweden. Thromb Res 2015;136:216-20 